\title{
ELSA - training the next generation of space astrometrists
}

\section{Lindegren ${ }^{1}$, A. Bijaoui ${ }^{2}$, A. G. A. Brown ${ }^{3}$, R. Drimmel ${ }^{4}$, L. Eyer ${ }^{5}$, S. Jordan ${ }^{6}$ M. Kontizas ${ }^{7}$, F. van Leeuwen ${ }^{8}$, K. Muinonen ${ }^{9}$,

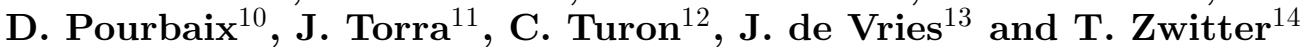

${ }^{1}$ Lund Observatory, Lund University, Sweden, email: lennart@astro.lu.se

${ }^{2}$ Observatoire de la Côte d'Azur, Nice, France, email: albert.bijaoui@obs-nice.fr

${ }^{3}$ Leiden Observatory, Leiden University, The Netherlands, email: brown@strw.leidenuniv.nl

${ }^{4}$ Istituto Nazionale di Astrofisica, Torino, Italy, email: drimmel@oato.inaf.it

${ }^{5}$ Geneva Observatory, Switzerland, email: Laurent.Eyer@obs.unige.ch

${ }^{6}$ Astronomische Rechen-Institut, Heidelberg, Germany, email: jordan@ari.uni-heidelberg.de

${ }^{7}$ Dpt. of Astrophysics, Astronomy and Mechanics, Athens, Greece, email: mkontiza@cc.uoa.gr

${ }^{8}$ Institute of Astronomy, Cambridge, United Kingdom, email: fvl@ast.cam.ac.uk

${ }^{9}$ Observatory, Univ. Helsinki, Finland, email: karri.muinonen@helsinki.fi

${ }^{10}$ Institute of Astronomy and Astrophysics, Brussels, Belgium, email: pourbaix@astro.ulb.ac.be

${ }^{11}$ Departament d'Astronomia i Meteorologia, Barcelona, Spain, email: jordi@am.ub.es

${ }^{12}$ Observatoire de Paris, Meudon, France, email: catherine.turon@obspm.fr

${ }^{13}$ Dutch Space B. V., Leiden, The Netherlands, email: j.de.vries@dutchspace.nl

${ }^{14}$ Faculty of Mathematics and Physics, Ljubljana, Slovenia, email: tomaz.zwitter@fmf.uni-lj.si

\begin{abstract}
ELSA (European Leadership in Space Astrometry) is an EU-funded research project 2006-2010, contributing to the scientific preparations for the Gaia mission while training young researchers in space astrometry and related subjects. Nine postgraduate $(\mathrm{PhD})$ students and five postdocs have been recruited to the network. Their research focuses on the principles of global astrometric, photometric, and spectroscopic measurements from space, instrument modelling and calibration, and numerical analysis tools and data processing methods relevant for Gaia.
\end{abstract}

Keywords. astrometry, instrumentation: detectors, methods: data analysis, methods: numerical, space vehicles, techniques: photometric, techniques: radial velocities

\section{Background}

The experience and success of the Hipparcos mission, launched in 1989 by the European Space Agency (ESA), have made space astrometry into a specialty of Europe. This was emphasised by the selection, in 2000, of the Gaia project as one of the major future missions within ESA's scientific programme. Gaia builds directly on the specific know-how accumulated in Europe during the Hipparcos era. Many of the scientists involved in defining Gaia gained their experience with Hipparcos 20-30 years ago, and are now approaching retirement, or are already retired. A new generation of scientists must therefore take the lead into the future. Their expertise is needed both to formulate new projects and to make proper use of the vast output of Gaia and other large-scale surveys. ELSA (European Leadership in Space Astrometry) was formed partly in response to the need to foster the development of a new generation of scientists in this research area.

ELSA is a Marie Curie Research Training Network (RTN) supported by the European Community's Sixth Framework Programme (FP6). The purpose of an RTN is to allow 
research teams to implement a structured training programme for young researchers in the context of a well-defined collaborative research project. In our case this project is part of the scientific preparations for the Gaia mission, and therefore strongly linked to - but not formally part of - the Gaia Data Processing and Analysis Consortium. The ELSA contract covers four years (2006-2010), has a budget of 2.8 MEuro and involves 14 partners with Lund University as coordinator (see list of authors).

\section{Research project}

The scientific objective of ELSA is to develop theoretical understanding and practical analysis tools of importance for the Gaia mission. The title emphasizes astrometry, but this term should be understood in a wide sense including the measurement of the complementary (and strongly interrelated) photometric, spectroscopic, orbital, and structural properties of the objects possible with a scanning space observatory such as Gaia.

The project focuses on certain aspects of the mission where a number of common methods are applied to address the problems and develop new tools:

- Space astrometry: Global methods of space astrometry in the framework of General Relativity; sources of systematic errors in Gaia and their effects on the final results; interdependence of astrometric, photometric, and radial velocity information for space astrometry missions, including requirements for ground-based standards.

- Instrument modelling: CCD radiation damage effects on astrometry, photometry, and radial velocity; chromaticity effects; attitude modelling.

- Numerical analysis: Scaled-down model solutions; methods for detection and management of outliers; impact of weighting schemes on accuracy and convergence; alternative solution methods.

- Data processing: Implementation and optimisation of parallel super-computing and GRID technology for Gaia data processing and instrument simulation; data processing methods for the analysis of complex sources and irregular time series observations.

\section{Implementation}

The network has appointed nine postgraduate $(\mathrm{PhD})$ students and five postdocs. To take up their positions the fellows have to move to a new country. The PhD students are enrolled in the regular postgraduate programme at their respective host institute, but also participate in a number of network activities designed to promote collaboration, mobility, and training of project-related and general skills. The role of the postdocs is to carry out research, add specific expertise and transfer knowledge within the network.

As part of these activities, a series of network-wide meetings are organised with participation from the science community. The first one is the ELSA School on the Science of Gaia held in Leiden, 19-28 November 2007, and the final one will be the ELSA Conference on the Simulation and Analysis of Space Astrometry Data, to be held in Paris (planned for May 2010). In between, there will be two schools or workshops on more technical matters. For more and updated information, see http://www.astro.lu.se/ELSA.

\section{Acknowledgements}

The Marie Curie Research Training Network ELSA is supported by the European Community's Sixth Framework Programme under contract MRTN-CT-2006-033481. 